Original article

\title{
Urodynamic values role for females with different types of urinary incontinence
}

\author{
Zane Pilsetniece, Egils Vjaters
}

Pauls Stradins Clinical University Hospital, Riga, Latvia

Received 24 May 2020, Revised 2 May 2021, Accepted 27 May 2021

(C) 2020, Russian Open Medical Journal

\begin{abstract}
Aim - The aim of this study was to analyse how conventional urodynamic values differ between females with specific types of urinary incontinence (UI).

Material and Methods - Cross-sectional study enrolled 666 females with UI. Based on patient history and questionnaires they were divided into three groups: stress (SUI), mixed (MixUI) and urgency (UUI). Physical investigation and urodynamics were performed. The continuous factors: age and urodynamic data were evaluated using Multinomial regression and ANOVA test using SUI, MixUI, UUI as outcome groups.

Results - Analysing urodynamic parameters significant difference between at least two groups was shown by the cystometric capacity and maximum flow rate: both highest in the SUI group; residual urine, opening detrusor pressure, maximum urethral closure pressure at rest, functional urethral length at rest: all highest in the UUI group. Mainly all urodynamic data showed significant difference between SUI/UUI, and MixUI/UUI groups, while difference between SUI/MixUI were not significant.

Conclusions - Most of urodynamic data for MixUI group patients do not differ from SUI group. UDS parameters like: maximum flow rate, residual urine, opening detrusor pressure, maximum urethral closure pressure at rest, functional urethral length at rest can help to distinguish SUI and MixUI groups from UUI group.
\end{abstract}

Keywords: Female, Pressure-flow study, Urinary incontinence, Urodynamics, Urethral pressure profilometry.

Cite as Pilsetniece Z, Vjaters E. Urodynamic values role for females with different types of urinary incontinence. Russian Open Medical Journal 2021; 10: e0316.

Correspondence to Zane Pilsetniece. Address: Urological Department, Pauls Stradins Clinical University Hospital, Pilsonu str 13, Riga, LV1002, Latvia. Phone: +371 29119432. Fax: +37167069952. Email: zpilsetniece@gmail.com.

\section{Introduction}

A key factor during the planning of therapy for female patients with urinary incontinence (UI) is the specific type of UI [1, 2]. Three major types of UI are typically distinguished: stress $\mathrm{UI}$ (SUI), urgency UI (UUI), and mixed UI (MixUI). The type of UI can be determined on the basis of medical history, patient questionnaires, physical examination, and in some cases urodynamic study (UDS) is also indicated [1, 3-5].

Another factor of importance for planning the therapy of $\mathrm{UI}$ is that the complaints of $\mathrm{UI}$ may be accompanied by some other lower urinary tract symptoms (LUTS), which may change the optimum mode of treatment. Currently UDS provides the most accurate method for objectively determining the type of lower urinary tract dysfunction, especially such conditions as impaired sensitivity and contractility of detrusor, or possible infravesical obstruction [6].

Even though UDS is regarded as the gold standard of LUTS diagnostics, the value of these tests for distinguishing various UI types in female patients is still disputed. Furthermore, no Level 1 studies have demonstrated the clinical value of UDS for patients with UI [3].

It has been previously claimed that the risk factors for unsuccessful therapy of SUI in female patients is intrinsic sphincter deficiency (ISD), detrusor overactivity (DO) and voiding dysfunction, while the need for further studies was emphasized [7].

When considering the recommendations from different guidelines review for female patients with UI, it is clear that UDS can provide valuable additional information that is relevant to each individual, but important questions remain: At what stage of examination should UDS be performed, for which patients, and how can the results of UDS help to predict the outcomes of therapeutic interventions [8]? Two non-inferiority, multicentre, randomized, controlled trials demonstrate that preoperative UDS don't improve outcomes of SUI surgery [9]. At the same time, guidelines emphasize that UDS is indicated for patients with recurrent $\mathrm{UI}$ after surgery $[1,8]$. However, in case of recurrent $\mathrm{UI}$ after surgical treatment, comparing UDS before/after surgery could help analyse the group for which treatment has not been effective and thus eventually changing the tactics or at least informing the patient about statistically lower treatment effectiveness.

Taking into account the aforementioned considerations, the major objective of this study was to establish whether there are substantial differences in urodynamic parameters between various categories of $\mathrm{UI}$ patients, which were established through patient history, questionnaires and physical examination. 


\section{Material and Methods}

\section{Study design}

The enrolment in this cross-sectional study included 666 females with UI who were referred to the functional diagnostics department for urodynamic testing. All patients were interviewed and examined by the same physician, including taking the medical history and vaginal examination (evaluating for urethral hypermobility and pelvic organ prolapse according to the BadenWalker half way system). All of the included patients were able to complete on their own the questionnaires: Urogenital Distress Inventory - Short form (UDI-6), International Consultation on Incontinence Questionnaire - Urine incontinence (ICIQ-UI). On the basis of patient history, physical evaluation and questionnaires, the patients were divided into three groups: SUI, MixUI, UUI (International Continence Society urinary incontinence definitions were used: SUI is the complaint of involuntary leakage on effort or exertion, or on sneezing or coughing. UUI is the complaint of involuntary leakage accompanied by or immediately preceded by urgency. MixUI is the complaint of involuntary leakage associated with urgency and also with exertion, effort, sneezing, or coughing) [10].

\section{Urodynamic technique}

For all UDS tests MMS-Solar Silver waterfilled equipment was used. The following conventional urodynamics studies were performed: filling-cystometry followed by pressure-flow study (MCM) and urethral pressure profilometry (UPP). A triple lumen 9 Fr UDS catheter was used.

UPP was performed in supine position, with catheter pulling rate of $2 \mathrm{~mm} / \mathrm{s}$, filling rate of $2 \mathrm{~mL} / \mathrm{min}$. Both resting and cough stress profiles were analysed during UPP (the cough test was repeated at least three times during the stress profile).

MCM was performed in sitting position. The filling rate was 50 $\mathrm{mL} / \mathrm{min}$ and was reduced to $30 \mathrm{~mL} / \mathrm{min}$ if the first sensations were reported before the bladder was filled to $100 \mathrm{ml}$. After filling every $100 \mathrm{ml}$ into the bladder, the patient was asked to repeat strong cough test 3 times in a row. Reaching cystometric capacity patient urinated in privacy.

\section{Statistical analysis}

The descriptive statistics were calculated as count/ratio for categorical variables and mean values with standard deviation to illustrate variation. The differences of continuous factors: age and urodynamic data (cystometric capacity (CC), maximal urinary flow from Pressure-flow study (Qmax), calculated residual urine after pressure flow study (RU), opening detrusor pressure ( $p_{\text {det_open), }}$ maximal urethral closure pressure at rest (MUCP_rest), maximal urethral closure pressure during cough stress (MUCP_cough_stress), functional urethral length at rest (FUL_rest), functional urethral length during cough stress (FUL_stress), pressure transmission ratio (PTR)) were evaluated using ANOVA tests using SUI, MixUI, and UUI as the outcome groups and Bonferroni correction for multiple comparisons. The impact of factors on belonging to one of the analysed groups was assessed using the multinominal regression (analysing OR of the factors).

\section{Results}

Patient distribution according to the UI type and age is shown in Table 1.

Among the analysed UDS parameters, a significant difference between at least two groups was shown by the CC, which was highest in the SUI group; the $Q_{\text {max }}$ : highest in the SUI group; RU: highest in the UUI group; $p_{\text {det }}$ open, MUCP_rest, FUL_rest: all highest in the UUI group.

As shown in Table 2, in the majority of comparisons, a significant difference was observed specifically between the SUI/UUI groups and between the MixUI/UUI groups. We observed a significant difference between the SUI and MixUI groups only according to the CC parameter. None of the analysed stress profile measurements (MUCP_cough_stress, FUL_cough_stress, and PTR) showed a significant difference between any of the groups.

Dividing the individual group of patients for whom neither SUI nor UUI were registered during the UDS, results show that there was a total of 297 such patients. Accordingly: SUI 74 (50.3\% of all SUlgroup), MixUI 171 (40.1\% of all MixUlgroup), UUI 52 (55.9\% of all UUIgroup). After the Chi-test the failure to register $\mathrm{UI}$ is a significant indicator for categorising a woman with UI complaints in one of the subgroups above $(p=0.006)$. When analysed alone patients not having urodynamically confirmed stress UI (SUI UDS) nor detrusor overactivity (DO) showed a lower OR for MixUI (0.61, $p<0.01)$, while having a higher OR for UUI $(1.70, p=0.02)$. This value did not show statistically significant impact on SUI group $(p=0.11)$.

When analysed alone, patients having DO during the filling phase showed a lower OR for the SUI group (OR=0.34, $p<0.01)$, while the OR values were higher in UUI group $(O R=1.98, p<0.01)$. Thus, the presence of DO is likely to include patients in the UUI group and to reduce the presence of SUI. Also, when analysed alone, the patients with SUI_UDS showed lower OR for UUI group $(O R=0.11, p<0.01)$, while having higher $O R$ for $S U I$ group ( $O R=1.48$, $p=0.04)$ and MixUI group (OR=1.57, $p=0.01)$. Because the values of SUI and MixUI groups are close, it could be concluded that if SUI_UDS is registered for MixUI patients, the SUI type is likely to prevail. The association of DO and SUI_UDS with specific urinary incontinence groups is shown in Table 3 and 4.

In our analysis of patients with registered both SUI_UDS and DO, only 25 patients were reported these both values $(3.4 \%$ of all SUI, $4.7 \%$ of all MixUI and $0 \%$ of all UUI group).

\section{Discussion}

The main aim of this study was to investigate whether conventional UDS values are different for women with different types of UI complaints. This could potentially be clinically relevant when planning UI therapy.

Table 1. Patient distribution according to the urinary incontinence type and age

\begin{tabular}{|c|c|c|c|c|c|c|}
\hline \multirow{3}{*}{ Age group } & \multicolumn{6}{|c|}{ Urinary incontinence (UI) type } \\
\hline & \multicolumn{2}{|c|}{$\begin{array}{l}\text { Stress UI } \\
147(22 \%)\end{array}$} & \multicolumn{2}{|c|}{$\begin{array}{l}\text { Mixed UI } \\
426(64 \%)\end{array}$} & \multicolumn{2}{|c|}{$\begin{array}{c}\text { Urgency UI } \\
93(14 \%)\end{array}$} \\
\hline & Count & $\mathrm{N} \%$ & Count & $\mathrm{N} \%$ & Count & $\mathrm{N} \%$ \\
\hline $18-44$ & 31 & 21.09 & 50 & 11.74 & 10 & 10.75 \\
\hline $45-64$ & 84 & 57.14 & 204 & 47.89 & 32 & 34.41 \\
\hline $65-85$ & 32 & 21.77 & 172 & 40.38 & 51 & 54.84 \\
\hline
\end{tabular}


Table 2. Urodynamic data with the mean and $\mathrm{p}$ values for different urinary incontinence groups

\begin{tabular}{|c|c|c|c|c|c|c|}
\hline & SUI Mean $\pm S D$ & MixUI Mean $\pm S D$ & UUI Mean $\pm S D$ & $P$ SUI/MIX & P SUI/UUI & $P M I X / U U I$ \\
\hline $\mathrm{CC}, \mathrm{ml}$ & $357.64 \pm 138.22$ & $284.13 \pm 141.65$ & $278.29 \pm 132.3$ & $<0.001$ & $<0.001$ & 0.922 \\
\hline Qmax, ml/sec & $21.04 \pm 8.96$ & $19.6 \pm 9.75$ & $13.93 \pm 8.14$ & 0.225 & $<0.001$ & $<0.001$ \\
\hline $\mathrm{RU}, \mathrm{ml}$ & $7.09 \pm 33.58$ & $10.42 \pm 51.51$ & $40.29 \pm 82.37$ & 0.639 & 0.001 & 0.003 \\
\hline p_det_open, $\mathrm{cmH} 2 \mathrm{O}$ & $21.29 \pm 14.41$ & $21.85 \pm 13.78$ & $27.53 \pm 16.67$ & 0.909 & 0.009 & 0.007 \\
\hline MUCP_rest, cmH2O & $63.51 \pm 32.21$ & $62.57 \pm 33.27$ & $74.07 \pm 35.97$ & 0.950 & 0.055 & 0.014 \\
\hline FUL_rest, mm & $33.55 \pm 12.19$ & $32.87 \pm 12.02$ & $36.41 \pm 10.06$ & 0.824 & 0.117 & 0.009 \\
\hline MUCP_cough_stress, $\mathrm{cm} \mathrm{H} 20$ & $75.37 \pm 39.34$ & $77.22 \pm 41.66$ & $85.24 \pm 45.07$ & 0.876 & 0.190 & 0.256 \\
\hline PTR, \% & $59.73 \pm 35.49$ & $62.8 \pm 35.81$ & $70.55 \pm 36.7$ & 0.633 & 0.062 & 0.153 \\
\hline
\end{tabular}

CC, cystometric capacity; Qmax, maximal urinary flow from Pressure-flow study; RU, calculated residual urine after pressure flow study;

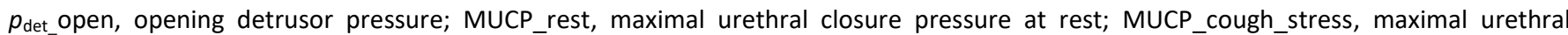
closure pressure during cough stress; FUL_rest, functional urethral length at rest; FUL_stress, functional urethral length during cough stress; PTR, pressure transmission ratio; SUI, Stress urinary incontinence group; MixUI, mixed urinary incontinence group; UUI, urgency urinary incontinence group.

Table 3. The association of detrusor overactivity (DO) with specific urinary incontinence groups: stress urinary incontinence (SUI), mixed urinary incontinence (MixUI), urgency urinary incontinence (UUI).

\begin{tabular}{|c|c|c|c|c|c|c|}
\hline & \multicolumn{2}{|l|}{ SUI } & \multicolumn{2}{|c|}{ MixUI } & \multicolumn{2}{|c|}{ UUI } \\
\hline & OR $(95 \% \mathrm{Cl})$ & $\mathrm{p}$-value & OR $(95 \% \mathrm{Cl})$ & $\mathrm{p}$-value & OR $(95 \% \mathrm{Cl})$ & $\mathrm{p}$-value \\
\hline no & 1* & & 1* & & 1* & \\
\hline $\mathrm{DO}_{\text {yes }}$ & $\begin{array}{c}0.34 \\
(0.20 \ldots 0.58)\end{array}$ & $<0.01$ & $\begin{array}{c}1.32 \\
(0.91 \ldots 1.92)\end{array}$ & 0.14 & $\begin{array}{c}1.98 \\
(1.25 \ldots 3.14)\end{array}$ & $<0.01$ \\
\hline
\end{tabular}

*Reference category.

Table 4. The association of urodynamically confirmed stress urinary incontinence (SUI_UDS) with specific urinary incontinence groups: stress urinary incontinence (SUI), mixed urinary incontinence (MixUI), urgency urinary incontinence (UUI).

\begin{tabular}{|c|c|c|c|c|c|c|}
\hline & \multicolumn{2}{|c|}{ SUI } & \multicolumn{2}{|c|}{ MixUl } & \multicolumn{2}{|c|}{ UUI } \\
\hline & OR $(95 \% \mathrm{Cl})$ & $\mathrm{p}$-value & OR $(95 \% \mathrm{Cl})$ & $\mathrm{p}$-value & OR $(95 \% \mathrm{Cl})$ & $\mathrm{p}$-value \\
\hline no & 1* & & 1* & & 1* & \\
\hline UDS yes & $\begin{array}{c}1.48 \\
(1.02 \ldots 2.16)\end{array}$ & 0.04 & $\begin{array}{c}1.57 \\
(1.11 \ldots 2.22)\end{array}$ & 0.01 & $\begin{array}{c}0.11 \\
(0.05 \ldots 0.26)\end{array}$ & $<0.01$ \\
\hline
\end{tabular}

*Reference category.

Even though several previous studies of similar design failed to provide reliable justification for the clinical application of UDS [2] [4] [3], our data show a remarkable trend, where the majority of UDS parameters have statistically significant differences between the SUI/UUI and MixUI/UUI groups, while significant differences between the SUI/MixUI groups were less often observed. Also in the literature there are previously done studies which support conventional UDS to be able to make the most accurate diagnosis of UI type before starting the therapy [11]. Even though there are authors who say, "Conventional UDSs have been criticized for being nonphysiological tests, and there is disagreement between the urodynamic and symptom-based diagnoses in 19-44\% of patients" [12], UDS is still recommended when the selected treatment has proven to be ineffective, or prior to invasive procedures $[1,5]$. However, it is important to understand the objectives of UDS, and to establish correlations between the UDS results and the optimal treatment options. None of the current guidelines provides a clear answer to these questions, while most of the publications recognize the need for further studies.

Our data indicate that UDS can provide additional information, which helps to differentiate between specific UI types in female patients.
The incidence of MixUI increases with age [13]. Still, a question remains about the pathogenic causes for this, such as infravesical outlet factors or dysfunction of detrusor muscle. According to our results, the parameters for MixUI cases were closer to SUI, therefore such cases may be rather viewed as SUI. At the same time, there is evidence that the risks of LUTS, including DO and detrusor hypocontractility, increase with age $[13,14]$. Thus, UDS may be of substantial benefit for older women with lower urinary tract symptoms, in order to select the appropriate therapy and to predict potential risks of drugs or surgery for the treatment of UI.

When performing UDS prior to planned surgical treatment of $\mathrm{UI}$, our main goal is to identify possible risk factors to unsuccessful outcome of treatment and to predict and reduce possible complications. This is closely associated with accurate determination of the lower urinary tract dysfunction type, as previously postulated by Rosier [3]. Ineffective treatment of SUI cannot always be attributed to the procedure itself, but also to inappropriate choice of treatment in each particular case, even if the UI type is correctly established. In other studies it is also mentioned that the UDS do not have any clinical and economical value for isolated SUI prior to surgery, but we have to bear in mind that analysed patient group in these studies was isolated SUI [9, $15,16]$. At the same time, UDS is highly recommended at a recurrent UI after surgical treatment. In such cases, I always ask myself if I have done everything to assess the potential risk factors for a recurrent $\mathrm{UI}$ before the surgery?

Different opinions have been expressed in previous studies regarding the clinical significance of UDS for predicting the outcomes of $\mathrm{UI}$ treatment. Our data indicate that $p_{\text {det_open, }}$ FUL_rest, and MUCP_rest, SUI_UDS, registered DO parameters show significant differences between the SUI and UUI groups. This means that the aforementioned parameters can be used for distinguishing between these UI types. At the same time, Pandey et al. reported that analysis of urethral profile had no clinical significance for planning surgical treatment of SUI [4]. A published review of this topic claims that female patients with low VLPP and MUCP showed less benefit from surgery and had higher recurrence of UI [17]. Another research group reports that the only UDS findings associated with negative TVT/TOT results were recorded DO episodes during the filling phase. [18] Contrary to Aydin et al., Fletcher and Lemack emphasized [7] that observation of DO during UDS for patients with clinical manifestation of SUI and planned surgical treatment did not predict inferior postoperative outcomes. Our results show that DO is significantly 
more common among patients of the UUI group compared to the SUI group, even though it is known that DO has been observed also for healthy individuals of the control group [6, 8]. With regard to the treatment of MixUl patients, women with subjective complaints of MixUI and UDS findings of DO, symptomatic pharmaceutical treatment of overactive bladder could be justified as first line of therapy. At the same time, as noted previously, we observed that the majority of UDS measurements show the most differences between the SUI/UUI and MixUI/UUI groups, and less between the SUI/MixUI groups. This may indicate that the treatment of MixUI patients with prevailing SUI symptoms, as well as recorded DO episodes, should mostly follow the guidelines for treatment of SUI.

Some publications indicate that conventional UDS may be justified even for isolated SUI, because it may provide additional information about lower urinary tract dysfunctions [3, 4]. In the opinion of McGuire, complete diagnostics of UI patient should include even video urodynamics test and VLPP because of the observation that weakened proximal urethral closure mechanism can result in UI episodes even at slight increase of $p_{\mathrm{abd}}$, despite completely normal function of urethral sphincter [19].

When discussing the clinical value of UPP, most often the attention is directed towards MUCP. However, the data about the correlation of MUCP with efficacy of surgical $U I$ treatment are inconclusive $[7,16]$. Our study has shown that the MUCP_rest and FUL_rest parameters enable reasonable differentiation of SUI and MixUI from the UUI group, which indicates that surgery of SUI could be probably recommended for the patients of the first two groups. The urethral rest profile measurements were the lowest for patients of the MixUI group. This observation of our study support the experts who believe in UPP usefulness for females with UI [20] but is in contrast with the reports that measurements of urethral profile do not provide reliable information for differentiation between different types of UI [3].

Taking into account all of the above, the question remains, whether UDS may be more cost-effective for the purpose of accurate diagnosis, compared to routine prescription of drugs for patients with mixed complaints.

\section{Conclusions}

Our study indicates that in most cases there is no statistically significant difference between the UDS results in patients of SUI and MixUl groups, but there is a significant difference between the SUI/UUI and MixUI/UUI groups. Therefore, the pathogenesis of SUI and MixUl conditions is more similar and can be distinguished from the UUI group with UDS parameters like: Qmax, RU, p_det_open, SUI_UDS, registered DO from filling cystometry and pressure-flow study and MUCP_rest and FUL_rest from urethral pressure profilometry. In our opinion, decisions regarding the clinical approach to MixUI cases are the least certain and difficult. Knowledge from this study may provide a substantial benefit for recommending the optimal diagnostic algorithm for MixUI patients.

\section{Study limitations}

The limitation of this study is lack of control group with continent females. Also, it is necessary to evaluate specific association of urodynamic values with treatment outcome for females with different types of urinary incontinence.

\section{Conflict of interest}

Conflict of Interest: Author Pilsetniece $\mathrm{Z}$ has received a speaker honorarium from Companys Bionorica, Olainfarm, Norameda, Zentiva. Author Vjaters E has received a research grant from Company Orion and speaker honorarium from Companys Orion, Ipsen, Bayer, Janssen.

\section{Ethical approva}

All procedures performed in studies involving human participants were in accordance with the ethical standards of the institutional and/or national research committee and with the 1964 Helsinki declaration and its later amendments or comparable ethical standards. The study was approved by The Research Ethics Committee of Pauls Stradins Clinical University Hospital Development Society / Nr 210813-17L

\section{References}

1. Abrams P, Andersson KE, Birder L, Brubaker L, Cardozo L, Chapple C, et. al. Fourth international consultation on incontinence recommendations of the international scientific committee: Evaluation and treatment of urinary incontinence, pelvic organ prolapse, and fecal incontinence. Neurourol Urodyn 2010; 29(1): 213-240. https://doi.org/10.1002/nau.20870.

2. Pandey D, Anna G, Hana O, Christian F. Correlation between clinical presentation and urodynamic findings in women attending urogynecology clinic. J Midlife Health 2013; 4(3): 153-159. https://doi.org/10.4103/0976-7800.118992.

3. Rosier PF. The evidence for urodynamic investigation of patients with symptoms of urinary incontinence. F1000Prime Rep 2013; 5: 8. https://doi.org/10.12703/p5-8.

4. Borges JB, Guarisi T, Camargo AC, Borges PC. Correlation between urodynamic tests, history and clinical findings in treatment of women with urinary incontinence. Einstein (Sao Paulo) 2010; 8: 437-443. English, Portuguese. https://doi.org/10.1590/s1679-45082010ao1611.

5. Lucas MG, Bosch RJ, Burkhard FC, Cruz F, Madden TB, Nambiar AK, et al. EAU guidelines on surgical treatment of urinary incontinence. Eur Urol 2012; 62(6): 1118-1129. https://doi.org/10.1016/j.eururo.2012.09.023.

6. Abrams P, Cardozo L, Khoury S, Wein A, eds. Incontinence, 5th ed. European Association of Urology. Paris: ICUD-EAU. 2013; 1981 p. https://www.ics.org/Publications/ICI 5/INCONTINENCE.pdf.

7. Fletcher SG, Lemack GE. Clarifying the role of urodynamics in the preoperative evaluation of stress urinary incontinence. ScientificWorldJournal $\quad 2008$; $\quad 8$ : $1259-1268$. https://doi.org/10.1100/tsw.2008.159.

8. Syan R, Brucker BM. Guideline of guidelines: urinary incontinence. BJU Int 2016; 117(1): 20-33. https://doi.org/10.1111/bju.13187.

9. Hickling DR, Steele SS. The role of preoperative urodynamics in stress urinary incontinence surgery. Can Urol Assoc J 2017; 11(6Suppl2): S113-S115. https://doi.org/10.5489/cuaj.4581.

10. Abrams P, Cardozo L, Fall M, Griffiths D, Rosier P, Ulmsten U, et al; Standardisation Sub-committee of the International Continence Society. The standardisation of terminology of lower urinary tract function: report from the Standardisation Sub-committee of the International Continence Society. Neurourol Urodyn 2002; 21(2): 167178. https://doi.org/10.1002/nau.10052.

11. Nitti VW. The prevalence of urinary incontinence. Rev Urol 2001; 3 Suppl 1(Suppl 1): S2-S6. https://pubmed.ncbi.nlm.nih.gov/16985992.

12. Yamanishi T, Sakakibara R, Uchiyama T, Hirata K. Role of urodynamic studies in the diagnosis and treatment of lower urinary tract $\begin{array}{llll}\text { symptoms. } & \text { Urol Sci 2011; 22: } & 120-128 .\end{array}$ https://doi.org/10.1016/i.urols.2011.08.007.

13. Valentini FA, Robain G, Marti BG. Urodynamics in Women from Menopause to Oldest Age: What Motive? What Diagnosis? Int Braz $J$ 
Urol 2011; 37(1): 100-107. https://doi.org/10.1590/s167755382011000100013 .

14. Shin YS, On JW, Kim MK. Effect of aging on urodynamic parameters in women with stress urinary incontinence. Korean J Urol 2015; 56(5): 393-397. https://doi.org/10.4111/kju.2015.56.5.393.

15. Housley SL, Harding C, Pickard R. Urodynamic assessment of urinary incontinence. Indian J Urol 2010; 26(2): 215-220. https://doi.org/10.4103/0970-1591.65392.

16. Weber AM. Is urethral pressure profilometry a useful diagnostic test for stress urinary incontinence? Obstet Gynecol Surv 2001; 56(11): 720-735. https://doi.org/10.1097/00006254-200111000-00024.

17. lancu G, Peltecu G. Predicting the Outcome of Mid-urethral Tape Surgery for Stress Urinary Incontinence Using Preoperative Urodynamics - A Systematic Review. Chirurgia (Bucur) 2014; 104(3): 359-368. https://pubmed.ncbi.nlm.nih.gov/24956342.

18. Aydin S, Arioğlu Aydin Ç, Ersan F. Prediction of Mid-Urethral Sling Failure with Clinical Findings and Urodynamics. Low Urin Tract Symptoms 2017; 9(2): 89-93. https://doi.org/10.1111/luts.12121.

19. Mcguire EJ. Pathophysiology of Stress Urinary Incontinence. Rev Urol

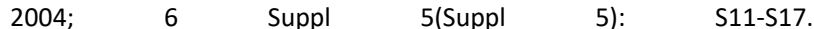
https://pubmed.ncbi.nlm.nih.gov/16985903.

20. Pizzoferrato AC, Fauconnier A, Fritel X, Bader G, Dompeyre P. Urethral Closure Pressure at Stress: A Predictive Measure for the Diagnosis and Severity of Urinary Incontinence in Women. Int Neurourol J 2017; 21(2): 121-127. https://doi.org/10.5213/inj.1732686.343.

Authors:

Zane Pilsetniece - Urologist, Department of Urology, Pauls Stradins Clinical University Hospital, Riga, Latvia. https://orcid.org/0000-0003-4094-0083.

Egils Vjaters - Professor of Urology, Department of Urology, Pauls Stradins Clinical University Hospital, Riga, Latvia. https://orcid.org/0000-0003-00543745. 